\title{
Internal carotid artery dissection presenting as hypoglossal nerve palsy
}

Ella Gordon ${ }^{1,2}$, Feda Fanadka ${ }^{2,3}$, Ahmad Atamna $^{1,2}$, Aviv Gour ${ }^{1,2}$, Belle Brahms-Tamir ${ }^{1,2}$, Hadeel Kinani $^{1,2}$, Nofar Frenkel-Manzur ${ }^{1,2}$, Natalie Shelly ${ }^{1,2}$, Roy Zaltzman ${ }^{1,2}$, Emily Elefant ${ }^{1,2}$, Meir Kestenbaum ${ }^{1,2}$ and Nirit Lev ${ }^{1,2 *}$

${ }^{1}$ Department of Neurology, Meir Medical Center, Israel

${ }^{2}$ Sackler Faculty of Medicine, Tel Aviv University, Israel

${ }^{3}$ Department of Radiology, Meir Medical Center, Israel

\begin{abstract}
Background: Carotid artery dissection is thought to be a consequence of a tear in the tunica intima, resulting in an intramural hematoma and creation of a false lumen. In such cases, thrombus formation and distal embolization may lead to ischemic stroke. Alternatively, the dissection plane separates the tunica media from the tunica adventitia, resulting in aneurysmal dilatation. This may result in compression of neighbouring structures. The clinical spectrum is diverse making the diagnosis illusive. Yet, carotid dissection is a main cause for strokes in young population, hence the importance of a timely diagnosis in order to minimize complications.

Case description: A 49-year-old man was admitted with slurred speech, left-sided head and neck ache and difficulty swallowing. He described an increasing difficulty moving his tongue to the left side. His symptoms began following a mild trauma. On examination, he had mild dysarthria and tongue deviation with difficulty to move the tongue to the left, findings characteristic of left hypoglossal nerve palsy. There was no evidence of Horner's syndrome or other focal neurological signs. CT brain scan was normal. Vascular imaging revealed left carotid artery dissection with significant mural hematoma.

Discussion and conclusions: Lower cranial nerves such as the hypoglossal nerve can be involved due to localized pressure in the neck. The Hypoglossal nerve passes close to the carotid artery hence may be compressed in carotid artery dissection, leading to hypoglossal nerve palsy. Carotid artery dissection accounts for a high proportion of stroke in young people. Carotid artery dissection is frequently associated with neck trauma, such as vehicle accidents, assaults, but can appear even in mild trauma. Usually, clinical suspicion of carotid dissection arises due to focal neurological deficits or Horner's syndrome. In the case described herein, hypoglossal nerve palsy due to local compression was the main clinical manifestation. When carotid artery dissection is suspected diagnosis through vascular imaging, and prompt treatment are imperative in order to prevent further complications.
\end{abstract}

\section{Introduction}

The hypoglossal nerve (CN XII palsy) is a pure motor nerve which controls both the intrinsic and extrinsic muscle of the tongue. Hypoglossal nerve palsy is a rare condition that impairs tongue movement, which may lead to dysarthria or dysphagia [1]. Neurological examination reveals atrophy of half of the tongue, with deviation and weakness of the tongue muscles in the affected side [1].

The hypoglossal nerve arises from a motor nucleus located in the ventral medulla oblongata, called the hypoglossal nucleus. The nerve exits the skull through the hypoglossal foramen. The extracranial part runs lateral to the carotid artery and anterior to the upper cervical spine, to reach the muscles of the tongue [2].

Carotid artery dissection is thought to be a consequence of a tear in the tunica intima, resulting in an intramural hematoma and creation of a false lumen. In such cases, thrombus formation and distal embolization may lead to ischemic stroke [3]. Alternatively, the dissection plane separates the tunica media from the tunica adventitia, resulting in aneurysmal dilatation. This may result in compression of neighbouring structures. The clinical spectrum is diverse making the diagnosis illusive. Yet, carotid dissection is a common cause for strokes hence the importance of a timely diagnosis in order to minimize potential morbidity and mortality [4].
We describe a case of isolated hypoglossal nerve palsy as a sole manifestation of carotid dissection.

\section{Case presentation}

A 49-year-old man was admitted to the emergency department with a 9-day history of slurred speech, left-sided ear and neck ache and difficulty swallowing. He described a progressive difficulty moving his tongue to the left. Symptoms developed after a mild trauma to his neck while he was riding a bicycle. He is generally healthy and physically active. He has a past medical history of hypertension treated with Ramipril 2.5 mg once a day.

On examination, there was mild deviation of the tongue to the left and difficulty to manipulate the tongue toward the left side, with mild atrophy of the left side of the tongue. There was no evidence of Horner's syndrome or involvement of other cranial nerves. The rest of his neurological examination did not reveal any pathological findings.

${ }^{\star}$ Correspondence to: Nirit Lev, Department of Neurology, Meir Medical Center, Kfar Saba, Israel, Tel: 972-504065316, E-mail: niritle@clalit.org.il

Key words: carotid artery dissection, hypoglossal nerve, vascular imaging

Received: October 05, 2020; Accepted: October 26, 2020; Published: October 29,2020 


\section{Investigations}

Blood tests including inflammatory makers, urea and electrolytes, liver function tests, blood count and coagulation studies were all unremarkable. Resting ECG showed sinus rhythm and no abnormalities.

Unenhanced brain computerized tomography (CT) scan was performed and was reported as normal. CT angiography (CTA) of the head and neck blood vessels demonstrated evidence of localized dissection (Figure 1) with mural hematoma (Figure 2 and Figure 3 ) in the left internal carotid artery. The hypoglossal canal proximity to the internal carotid artery is demonstrate in Figure 1.

During his admission to the neurological department, he suffered from continuous head and neck pain that gradually improved. He was treated by anticoagulation by subcutaneous Enoxaparin injections (Clexane $1 \mathrm{mg} / \mathrm{Kg}$ twice daily) followed by aspirin treatment.

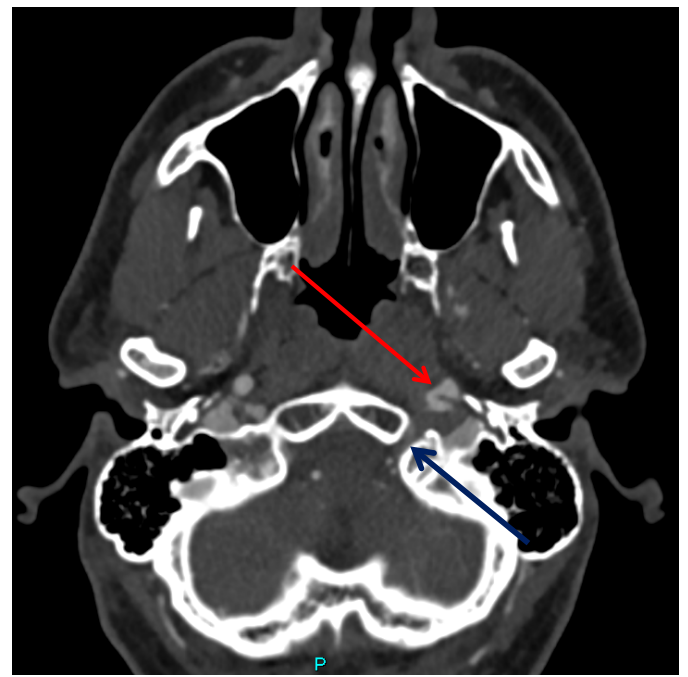

Figure 1. CT angiography axial images. Left internal carotid artery (LICA) dissection (red arrow) is demonstrated. The hypoglossal canal is marked with blue arrow.

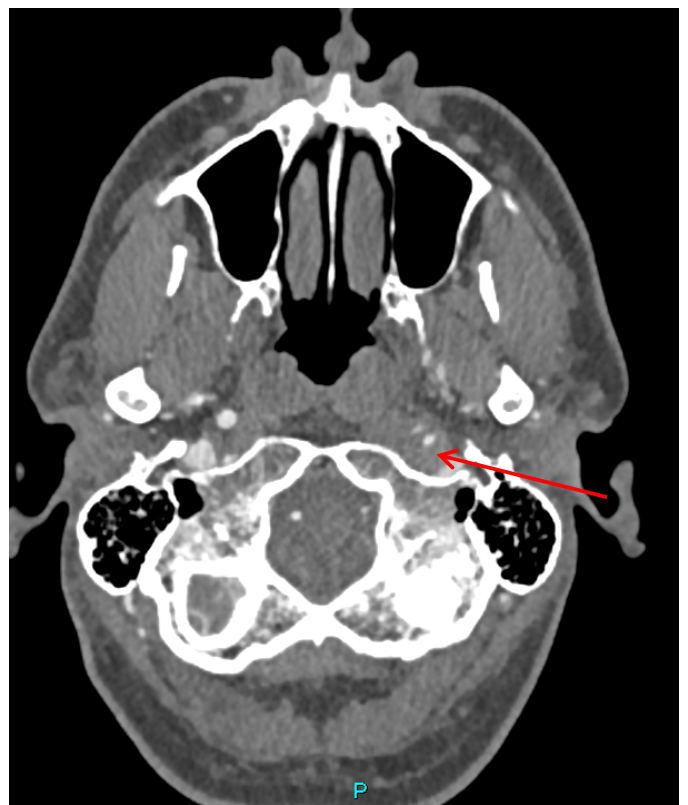

Figure 2. CT angiography axial images. Left internal carotid artery (LICA) narrowing of the true lumen due to mural thrombus (red arrow) is demonstrated.

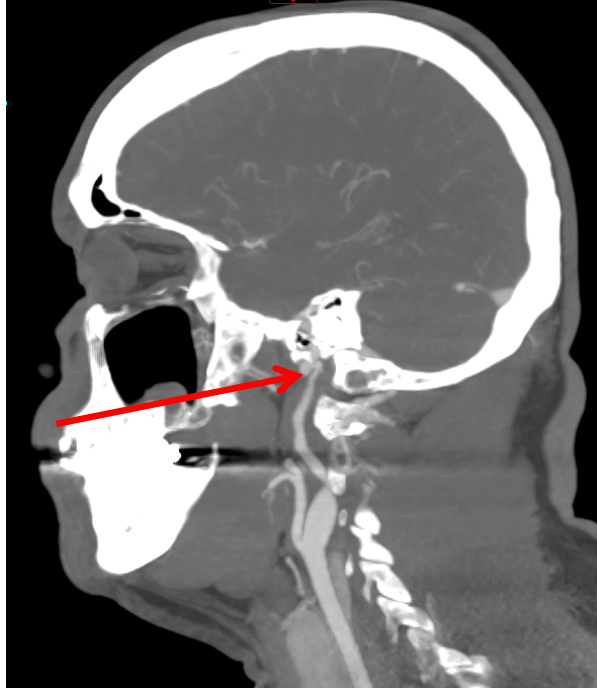

Figure 3. CT angiography sagittal MIP reformations demonstrating narrowing of the lumen of the distal extra-cranial LICA with mural thrombus (red arrow).

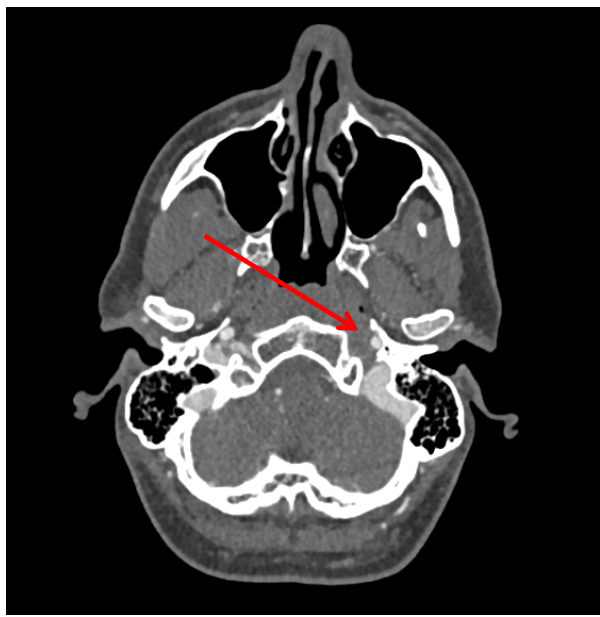

Figure 4. CT angiography axial images: Resolution of the narrowing of the true lumen of the LICA. A residual thrombus in the level of the dissection is demonstrated (red arrow).

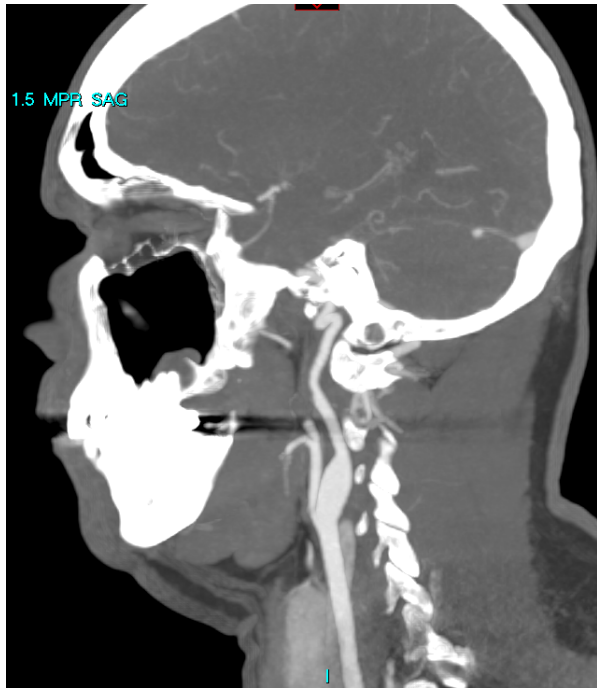

Figure 5. CT angiography sagittal MIP reformations demonstrating resolution of narrowing of the lumen of the distal extra cranial LICA. 
OUTCOME AND FOLLOW-UP: One month following his discharge the patient reported that his headaches resolved, his speech markedly improved and there was no tongue weakness. Follow-up CTA revealed improvement of the internal carotid artery lumen narrowing (Figure 4 and Figure 5).

\section{Discussion and conclusion}

We describe herein a 49-year-old man who was admitted due to a 9-day history of left head and neck pain accompanied by dysarthria, dysphagia, and tongue movement difficulties. Neurological examination revealed isolated left hypoglossal nerve palsy.

Isolated hypoglossal nerve damage is rare in clinical practice and is seen much less frequently than isolated damage to other cranial nerves such as the oculomotor, trochlear or abducens nerves [5,6]. Kean [7] reviewed 100 hypoglossal nerve palsy cases, accumulated through 26 years. The underlaying etiologies of hypoglossal nerve palsies included pressure due to tumors (49\%), cervical trauma (12\%), stroke $(6 \%)$, multiple sclerosis (6\%), surgical damage (5\%), Guillain-Barré syndrome (4\%), and infection (4\%) [7]. The largest series of 245 hypoglossal nerve palsies was published by Stino et al. [8]. They found that the common etiologic categories included: neoplastic $(42.2 \%)$, postoperative (29.3\%), idiopathic $(15.1 \%)$, inflammatory $(7.3 \%)$, radiation $(6.1 \%)$, and traumatic (4.1\%) [8]. Hence, localized pressure on the hypoglossal nerve was the leading cause for hypoglossal nerve palsy. Central nervous system (CNS) damage to the hypoglossal nucleus or supranuclear palsy can occur but are rarely causing isolated hypoglossal nerve palsy. This observation is corroborated by several other manuscripts [9-19].

During its course in the neck, the hypoglossal nerve passes in close proximity to the carotid artery, hence may be compressed in carotid artery dissection, leading to hypoglossal nerve palsy. In the case described herein, carotid dissection was the etiology for hypoglossal nerve palsy and the clinical picture. This is a rare cause for hypoglossal nerve palsy and was described previously only in a few case report [2024]. Moreover, this is a very rare manifestation of carotid dissection.

Carotid artery dissection accounts for a high proportion of stroke in young people $[3,4]$. Carotid artery dissection is frequently associated with neck trauma, such as assaults or vehicle accidents. It could appear even in mild trauma, for example, coughing, sneezing or neck manipulation. Focal neurological deficits or Horner's syndrome may be present. In the case described herein hypoglossal nerve involvement due to local compression was the main clinical manifestation, other than pain. When carotid artery dissection is suspected vascular imaging (CTA or MRA) should be done promptly. The Royal College of Physicians Stroke guidelines support the use of anticoagulation or antiplatelet therapy in the treatment of carotid artery dissection and the decision should be made according to the clinical features.

The case presented is exceptional since hypoglossal nerve palsy, although commonly caused by cervical pressure, is rarely caused by carotid dissection. Moreover, carotid dissection is most commonly manifested by strokes or Horner syndrome and not by local pressure. A high index of suspicion, early diagnosis, and identification of the cause with prompt initiation of appropriate therapy are mandatory in order to prevent further complications.

\section{References}

1. Shah A C, Barnes C, Spiekerman C F, Bollag LA (2015) Hypoglossal nerve palsy after airway management for general anesthesia: an analysis of 69 patients. Anesth Analg 120: 105-120. [Crossref]

2. Okunomiya T, Kageyama T, Suenaga T (2012) Teaching neuroimages: isolated hypoglossal nerve palsy due to internal carotid artery dissection. Neurol 79: 4. [Crossref]

3. Schievink WI (2001) Spontaneous dissection of the carotid and vertebral arteries. $N$ Engl J Med 344: 898-906.

4. Debette S, Leys D (2009) Cervical-artery dissections: predisposing factors, diagnosis, and outcome. Lancet Neurol 8: 668-678. [Crossref]

5. Fletcher A (2017) Persistent Isolated Unilateral Hypoglossal Nerve Palsy. Med Case Rep 3: 1 .

6. Shikino K, Noda K, Ikusaka M (2013) Transient Idiopathic isolated unilateral Hypoglossal nerve palsy. J Gen Intern Med 28: 591. [Crossref]

7. Keane JR (1996) Twelfth-nerve palsy. Analysis of 100 Cases. Arch Neurol 53: 561-566 [Crossref]

8. Stino AM, Smith BE, Temkit M, Reddy SN (2016) Hypoglossal nerve palsy: 245 cases. Muscle Nerve 54: 1050-1054. [Crossref]

9. Castling B, Hicks K (1995) Traumatic isolated unilateral hypoglossal nerve palsy-case report and review of the literature. Br J Oral Maxillofac Surg 33: 171-173.

10. Hong S, Lee J (2008) Isolated unilateral paralysis of the hypoglossal nerve after transoral intubation for general anesthesia. Dysphagia 4: 354-356. [Crossref]

11. Durrani F, Singh R (2009) Isolated hypoglossal nerve palsy due to infected impacted tooth. Case Rep Med 4: 231947. [Crossref]

12. Mahadevappa K, Chacko T, Nair A (2011) Isolated unilateral hypoglossal nerve palsy due to vertebral artery dissection. Clin Med Res 10: 127-130. [Crossref]

13. Hewett R, Stewart G PO (2011) 14 isolated hypoglossal nerve palsy caused by synovial cyst. J Neurol Neurosurg Psychiatr 82: 376-377. [Crossref]

14. Patron V, Roudaut P, Lerat J, Vivent M, Bessède J, et al. (2012) Isolated hypoglossal palsy due to cervical osteophyte. Eur Ann Otorhinolaryngol Head Neck Dis 129: 44 46. [Crossref]

15. Meila D, Wetter A, Brassel F, Nacimiento W (2012) Intermittent hypoglossal nerve palsy caused by a calcified persistent hypoglossal artery: an uncommon neurovascular compression syndrome. J Neurol Sci 323: 248-249. [Crossref]

16. Katsanos AH, Sioka C, Chondrogiorgi M, Papadopoulos A, Fotopoulos A, et al. (2018) Skull base metastasis revealed by bone scintigraphy in a patient with hypoglossal nerve palsy. Neurohospitalist 8: 188-190. [Crossref]

17. Rahsan I, Banu Ozen B, Ataman S, Ulk TB (2018) Hypoglossal nerve involvement in Lewis-Sumner syndrome: a case report. Acta Neurologica Belgica 118: 323-325. [Crossref]

18. Tomohiro W, Masato A, Yoshitaka M, Yuki N, Kaori S, et al. (2019) Hypoglossal Nerve Palsy as a Cause of Severe Dysphagia along with the Oropharyngeal Stenosis due to Occipitocervical Kyphosis. Case Reports Orthopedics: 7982847.

19. Ozaki K, Yamakami I, Higuchi Y, Fukutake T (2020) Isolated Hypoglossal Nerve Palsy Due to an Osteophyte with Atlantoaxial Dislocation. NMC Case Rep J 7: 201-204. [Crossref]

20. Mokri B, Silbert PL, Schievink WI, Piepgras DG (1996) Cranial nerve palsy in spontaneous dissection of the extracranial internal carotid artery. Neurol 46: 356-359. [Crossref]

21. Epstein E, Khan MA, Francis D, Sada P, Thuse M (2012) Carotid artery dissection causing hypoglossal nerve palsy. BMJ Case Reports: bcr0120125636. [Crossref]

22. Joshi P, Bourke D (2017) Isolated hypoglossal nerve palsy due to internal carotid artery dissection. Practical Neurol 17: 233-234.

23. Allingham W, Devakumar V, Herwadkar A, Punter M (2018) Hypoglossal Nerve Palsy Due to Internal Carotid Artery Dissection. Neurohospitalist 8: 199. [Crossref]

24. Chen Z, Jun Y, Hui L, Yuan C, Yin K, et al. (2019) Isolated hypoglossal nerve palsy from internal carotid artery dissection related to PKD-1 gene mutation. BMC Neurol 19: 276-281. [Crossref]

Copyright: (C2020 Gordon E. This is an open-access article distributed under the terms of the Creative Commons Attribution License, which permits unrestricted use, distribution, and reproduction in any medium, provided the original author and source are credited. 